\title{
Electrostatic effects in the apparent contact line region under a vapor bubble
}

\author{
Christiaan Ketelaar ${ }^{1}$ and Vladimir Ajaev2,3,a \\ ${ }^{1}$ Department of Mathematical Sciences, University of Delaware, Newark, DE 19716, USA \\ ${ }^{2}$ Department of Mathematics, Southern Methodist University, Dallas, TX 75275, USA \\ ${ }^{3}$ Kutateladze Institute of Thermophysics SB RAS, Lavrentyev Ave., 1, Novosibirsk, 630090, Russia
}

\begin{abstract}
Evaporation and local interface shapes are investigated in the apparent contact line region formed when an isolated vapor bubble is attached to a heated substrate. The model takes into account the electrostatic effects due to presence of ions in the liquid, as appropriate for water and aqueous solutions. For the apparent contact line region under a stationary bubble, local interface shapes are obtained for different parameter values. The model is then applied to the case of an expanding vapor bubble.
\end{abstract}

\section{Introduction}

Studies of heat transfer and viscous flow dynamics near apparent contact lines are important for a number of applications such as pool boiling, flow boiling, micro heat pipes, microfluidic actuation, and many others. Present work is motivated by a configuration of a vapor bubble on a heated substrate, as sketched in Fig. 1. Since pool boiling process starts with nucleation and growth of individual bubbles at the solid substrate, the configuration considered in the present study can provide important insights into the physics of boiling phenomena. Previous studies indicate that the part of the substrate under the bubble that appears dry on macroscale is often covered by a micro- or nanoscale adsorbed film. Thus, what appears to be a line of contact between the bubble surface and the dry substrate is in fact a transition region between the curved bubble surface and the flat ultra-thin film, as schematically shown in the figure. Suppression of evaporation in the ultra-thin film covering the heated substrate is explained by the action of the London-van der Waals dispersion forces. The transition region in this context is referred to as an apparent contact line. Numerous theoretical and experimental studies of evaporation near apparent contact lines are reviewed e.g. in Wayner [1] and Ajaev and Homsy [2]. While most of these studies address the situation when the apparent contact line is stationary, the approach can be extended to the case of moving contact lines as well, as was first shown by Ajaev et al. [3]. An important advantage of the apparent contact line approach is that it allows one to avoid dealing with a non-integrable shear-stress singularity encountered in other mathematical formulations. Such singularity leads to the unphysical conclusion about the resistance

\footnotetext{
a Corresponding author : ajaev@smu.edu
} 
force near the moving contact line being infinite; the approach of Ajaev et al. [3] allows one to relax the singularity and thus leads to finite value of resistance force at the contact line.

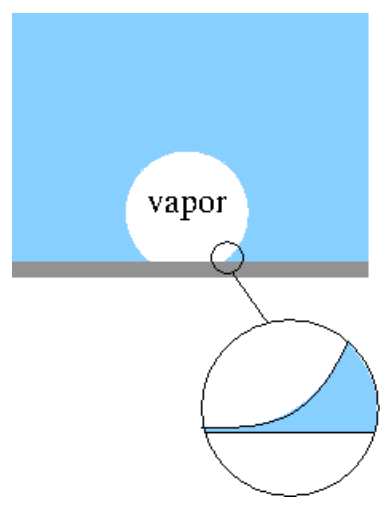

Figure 1. A sketch of a small isolated vapor bubble of radius $R_{0}$ formed on a heated substrate; the enlarged view of the apparent contact line region is shown.

The assumption of ultra-thin films being dominated by the London-van der Waals forces [1,2] is appropriate for describing experimental data with liquids such as pentane and hexane, but not in water and aqueous solution. The latter situation was investigated in the pioneering experimental study of Mazzoco and Wayner [4]. They considered evaporation of water and aqueous solutions of different concentrations. The general framework for mathematical modeling for this situation was developed in our previous work [5]. The objective of the present study is to use this framework to conduct additional parametric studies of the case of stationary contact line and then extend the approach to the case when the bubble is slowly expanding.

\section{Formulation}

Since the key novel aspect of our modeling approach is the addition of electrostatic effects, let us first discuss the model of electric field in the liquid. Liquid is assumed to be an aqueous solution, so electrical double layers of characteristic thickness equal to the Debye length are formed at interfaces. We use the standard definition of the Debye length, appropriate for 1:1 symmetric electrolyte solution at a temperature $T$,

$$
\lambda_{D}=\left(\frac{\varepsilon k_{B} T}{2 n_{0} e^{2}}\right)^{1 / 2}
$$

Here $e$ is the elementary charge, $k_{B}$ is the Boltzmann constant, $n_{0}$ is the equilibrium concentration of ions in the bulk, $\mathcal{E}$ is the dielectric permittivity. The electric field in the liquid is determined from the solution of the classical Poisson-Boltzmann equation,

$$
\nabla^{2} \psi=\kappa^{2} \sinh \psi
$$

We use $k_{B} T / e$ as the scale for the electric potential, so that the variable $\psi$ is nondimensional, with its value at the liquid-vapor interface denoted by $\tilde{\psi} ; \boldsymbol{K}$ is the ratio of the characteristic vertical length scale $d$ and the Debye length. Equation (2) is solved numerically with the conditions of 
constant scaled substrate potential (equal to -3 in the present study) and liquid-vapor interface charge density $q$; we use $\kappa=3, q=-0.04$ in all simulation results reported below.

Standard lubrication approximation $[6,7]$ is used for the viscous flow, with the assumptions of small capillary number and small ratio $d / R_{0}$. The film thickness (scaled by $d$ ) is then determined by the evolution equation,

$$
h_{t}+J+\frac{1}{3}\left[h^{3}\left(h_{x x}+\kappa^{2} Q \cosh \tilde{\psi}+\frac{\alpha}{h^{3}}\right)_{x}\right]_{x}-\frac{1}{2} \kappa Q q\left[h^{2} \tilde{\psi}_{h} h_{x}\right]_{x}=0
$$

Here the scaled evaporative flux is given by

$$
J=\frac{\delta\left(\frac{1}{2} \kappa^{2} Q q^{2}-h_{x x}-\kappa^{2} Q \cosh \tilde{\psi}-\frac{\alpha}{h^{3}}\right)+T_{0}}{K+h}
$$

The parameters $K, \delta$ arise from the nondimensional form of the so-called non-equilibrium condition at the interface, discussed e.g. in Ajaev and Homsy [2], $\alpha$ is the scaled Hamaker constant, $T_{0}$ is the nondimensional superheat. Detailed derivation of equations (3)-(4) can be found in our previous study [5]. Note that we account for the electrostatic contribution to stresses in the fluid at the interface not only in the normal stress, but also in the shear stress. The effects of London-van der Waals forces and the electrostatic interactions are added together in the formula for disjoining pressure, an approach that has an advantage due to its simplicity but also some limitations since in reality different components of disjoining pressure can be coupled together in a complicated manner. An alternative approach, free from these limitations, would be to consider potentials of intermolecular interactions which incorporate all relevant physics, as was done e.g. in Gatapova et al. [8].

Equation (3) with the expression for flux given by (4) in our approach has been solved numerically using a finite-difference approach for both steady and expanding/contracting bubbles.

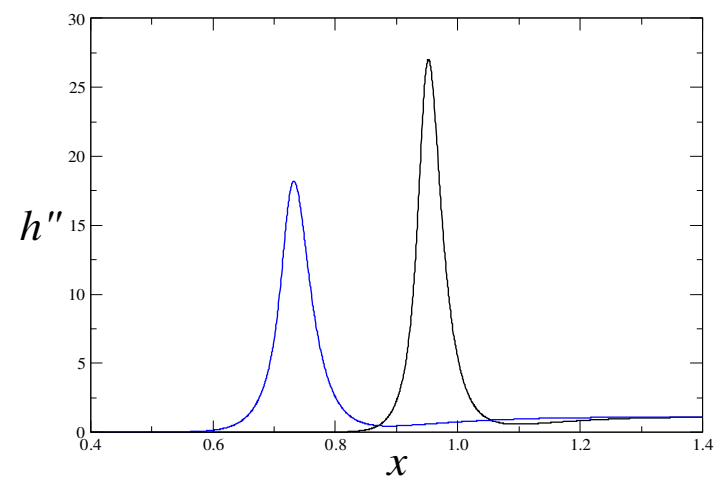

Figure 2. Interfacial curvature in the lubrication-type approximation as a function of local coordinate for $Q=$ $0.1, T_{0}=0.1, \delta=10^{-4}, \alpha=10^{-3}, K=0.1$ (black line) and $K=0.2$ (blue line).

\section{Results and discussion}

Changes in the interface shapes in the apparent contact line region are best visualized by plotting the curvature as a function of coordinate. Let us first discuss such plots for the case of steady bubbles. Figure 2 illustrates how the curvature profile changes with the non-equilibrium parameter, $K$. In our 
previous work, the value of this parameter was fixed at 0.1 , based on the assumption that the accomodation coefficient for evaporation at the interface is equal to unity [5]. However, in reality this coefficient can vary over a wide range, possibly several orders of magnitude, as discussed e.g. in the well-known paper of Marek \& Straub [9]. Thus, it is important to study the effect of variation of the accomodation coefficient on the interface shape; in our formulation such variation is equivalent to changes in $K$. Fig. 2 clearly shows that increasing this parameter leads to significantly smaller value of curvature and to widening of the profile, i.e increase in the width of the transition region between the macroscopic meniscus and the adsorbed film. Also, the apparent contact line shifts to the left. The decrease in the curvature can be explained by the fact the local evaporative flux, defined above by equation (4), is not as large as at the smaller $K$ and therefore the local curvature increase needed to maintain the steady-state configuration is less. Overall, the qualitative shape of the curvature profile remains the same, with a well-defined maximum in the transition region and a point of local minimum to the right from the location of the maximum.

For heat transfer applications, especially pool boiling, it is important to study the dynamics of contact line evaporation for bubbles which are expanding rather than steady. Such expansion results in a reduction of curvature away from the transition region with time. In our formulation, such curvature is the boundary condition at the right end point of the computational domain. To account for possibility of bubble expansion, we made this boundary condition time-dependent, with the constant rate of reduction of curvature equal to 0.01 in our nondimensional units. The initial curvature is set to unity. The resulting curvature profiles are shown in Fig. 3 at three different moments in time. Clearly, the location of the contact line region shifts to the left, but the shape of the profile does not change significantly, with just a small increase in the local curvature. This suggests that the local interface profile on that time scale quickly adjusts to the shape which is close to the steady one. In fact, the transient type behaviour can be seen during the initial stages of evolution, when the shape changes are slightly faster than in the quasi-steady regime corresponding to the blue and green lines in Fig. 3 .

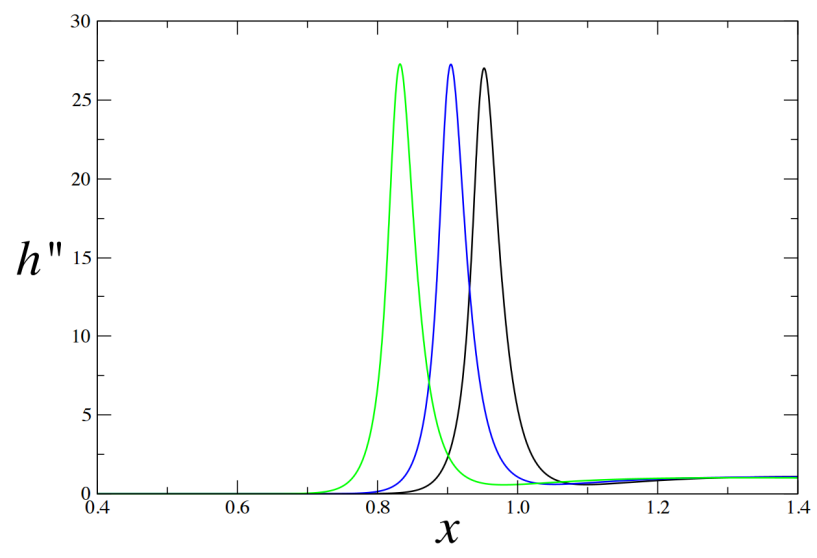

Figure 3. Local interfacial curvature profiles for the case of expanding bubble at time moments $t=0.1$ (black line), $t=5$ (blue line), and $t=10$ (green line) for $K=0.1$ and all other parameter values being the same as in Fig. 2.

The work was supported by the Russian Ministry of Education and Science (project identifier RFMEFI61314X0011). 


\section{References}

1. P.C. Wayner, AIChE J. 45, 2055 (1999)

2. V.S. Ajaev and G.M. Homsy, Annu. Rev. Fluid Mech., 38, 277 (2006)

3. V.S. Ajaev, G.M.Homsy, S. J. S. Morris, J. Colloid Interface Sci., 254, 346 (2002)

4. R.R.Mazzoco and P. C. Wayner, J. Colloid Interface Sci., 214, 156 (1999)

5. C. Ketelaar and V.S. Ajaev, Phys. Fluids , 27, 112110 (2015)

6. A. Oron, S.G.Bankoff and S.H. Davis, Rev. Mod. Phys., 69, 931 (1997)

7. R.V. Craster, O.K. Matar, Rev. Mod. Phys., 81, 1131 (2009)

8. E.Y.Gatapova, I.A. Graur, F. Sharipov, O.A. Kabov, Int. J. Heat and Mass Transfer, 83, 235 (2015)

9. R. Marek, J. Straub, Int. J. Heat Mass Transfer, 44, 39 (2001) 